\title{
Titan Atmospheric Entry Radiative Heating
}

Aaron Brandis and Brett Cruden

AMA Inc at NASA Ames, Moffett Field, California 


\section{Overview}

\section{Provide motivation and introduce EAST and computational tools}

Summary of Titan entry radiation literature

\section{EAST shock tube facility}

This presentation should convey 4 main points:

1) Data obtained in EAST for Titan entry

2) New data shows substantially larger radiance

3) Simulations agree significantly better with new data

4) Previously reported data can only be explained by contamination combined with other factors

Attempts were made to re-create this discrepancy by examining current facility operations and possible test gas contamination in previous experiments New EAST Titan data, Test 61, is available at httos://data.nasa.gov 


\section{Introduction}

- The joint NASA/ESA Cassini/Huygens mission resulted in significant efforts to understand radiative heating for Titan entry.

- Before arriving at Titan, there was significant uncertainty regarding the atmospheric composition. $\mathrm{CH}_{4}$ was suspected to be as high as $9 \%$ with Argon up to $20 \%$ (by mole).

- Cassini indicated that these numbers were closer to $2 \%$ for $\mathrm{CH}_{4}$ and $1 \%$ for Argon (by mole).

- Post flight simulations were conducted assuming a Boltzmann distribution of $\mathrm{CN}$ excited states

- If this were to be the case, Huygens may have burnt up during entry

- Consequently, experiments were performed in shock tubes and QSS/CR models developed. 


\section{Previous Works}

$5.15 \mathrm{~km} / \mathrm{s}, 98 \% \mathrm{~N}_{2}: 2 \% \mathrm{CH}_{4}, 0.1$ Torr, 400 - 430nm. EAST T43-25

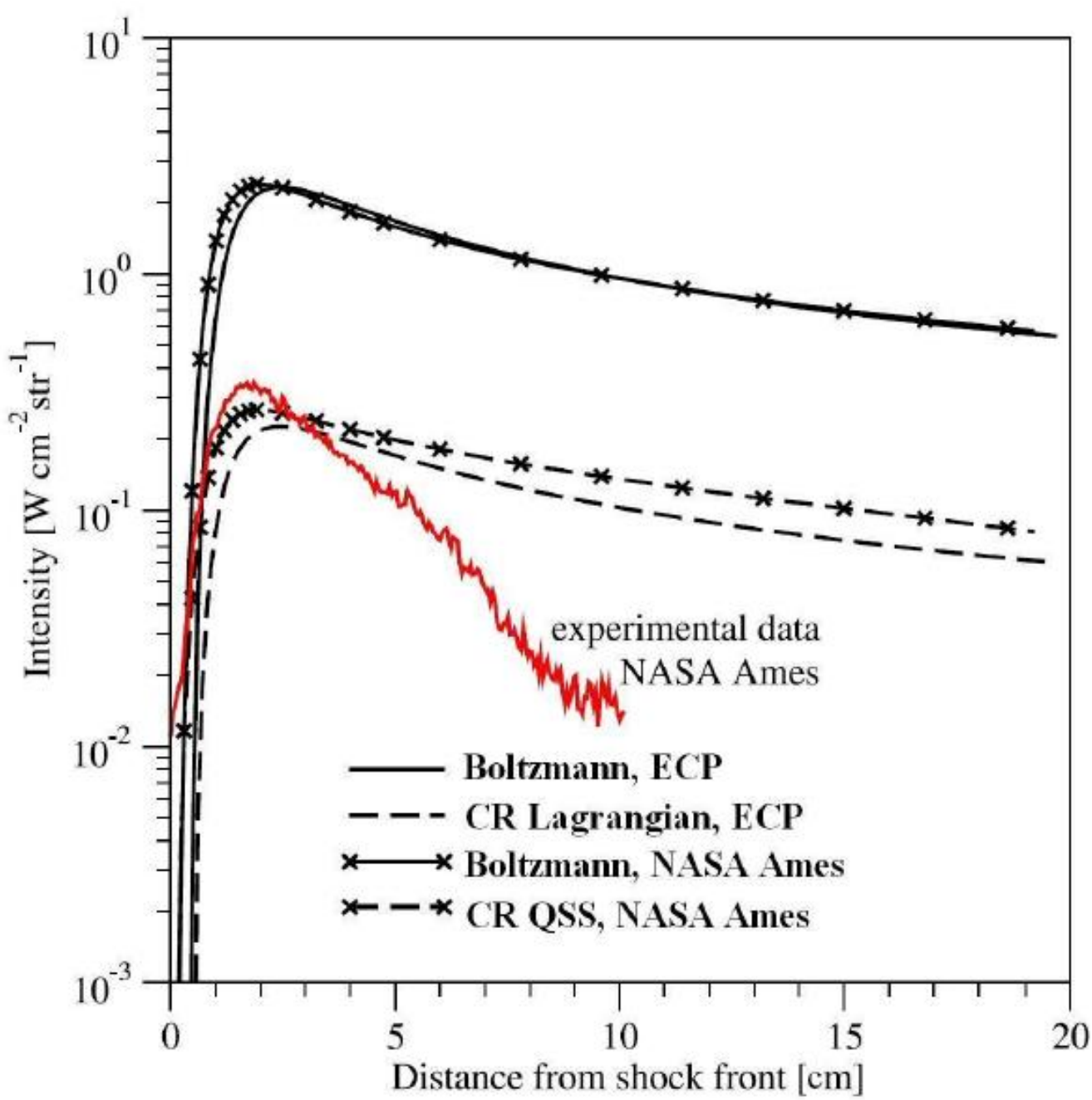

- Test 43 \& 45 from EAST (2003 to 2005)

- Boltzmann predictions shown to substantially over-predict

- CR models deemed to adequately match peak (within a factor of $\sim 2$ )

- Simulations showed slower decay rate than experiment

- X2 from Brandis \& Jacobs 


\section{Methodology}




\section{EAST Facility}

- EAST: Electric Arc Shock Tube, located at NASA Ames Research Center

- Shock is driven by an electric arc discharge.

- $10.16 \mathrm{~cm}$ in diameter at the test section.

- 4 spectrometers analyzing different spectral ranges in each shot. These ranges were typically:
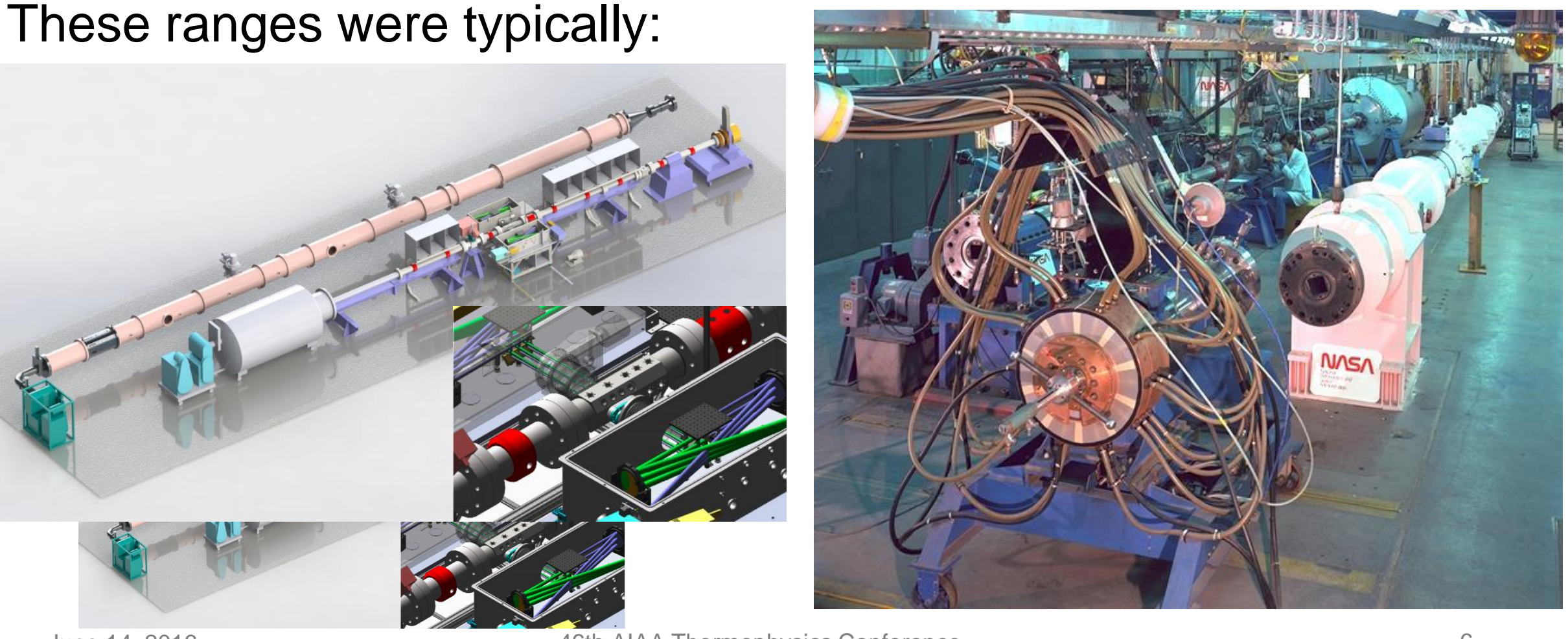


\section{Conditions}

-Huygens

—Fly By Candidate Trajectory 1

—Fly By Candidate Trajectory 3

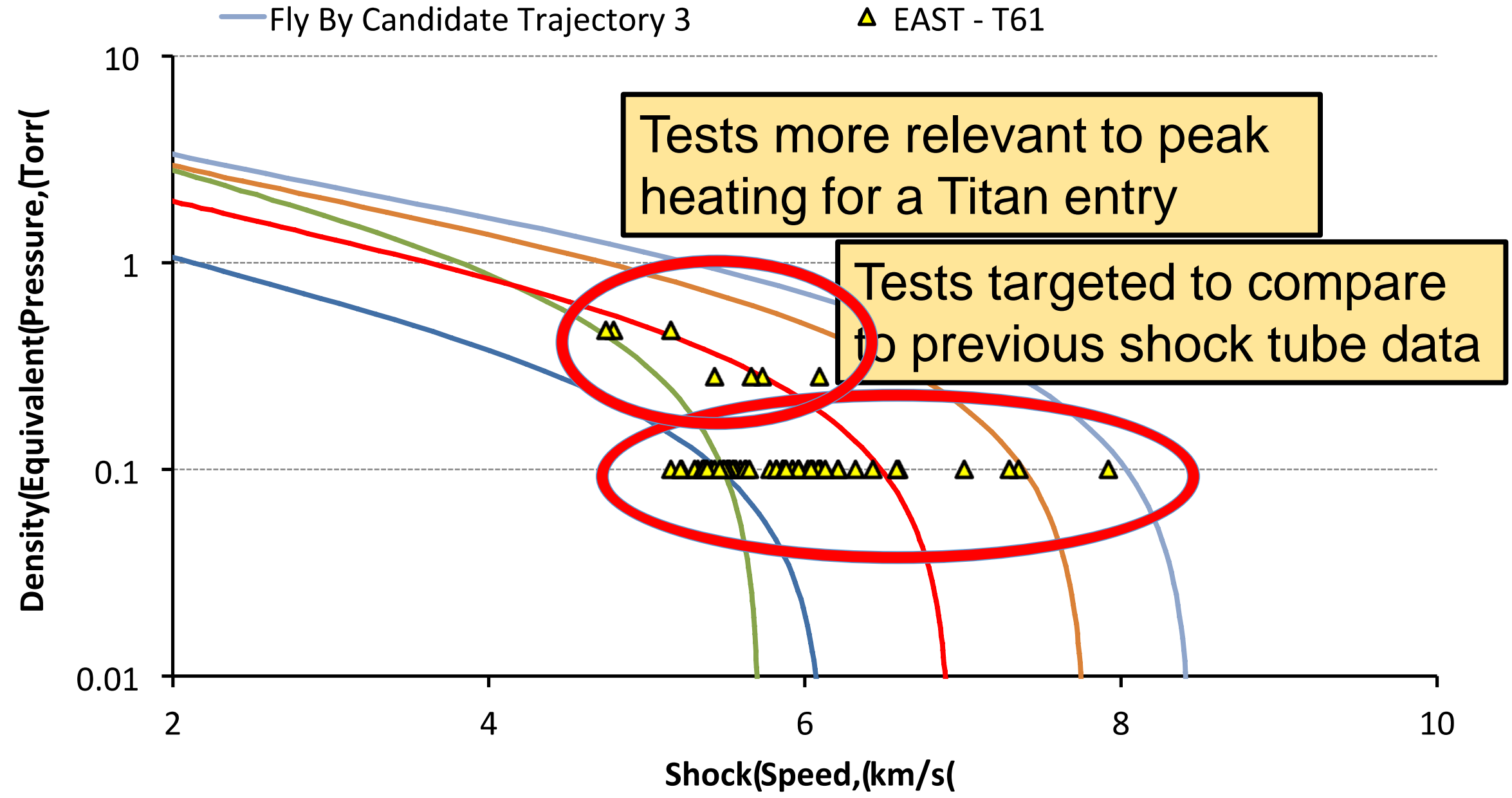




\section{DPLR and NEQAIR Simulations}

- Flowfield calculated with DPLR 4.04

- Two-dimensional grid of a $3 \mathrm{~m}$ sphere with 805 grid points on the stagnation line.

- The stagnation line being assumed analogous to the flow behind the shock in the EAST experiments.

- 21 species gas model.

- Radiation calculated with pre-release of NEQAIR v15

- CN non-Boltzmann model added in NEQAIR v14

- An over-prediction by atomic nitrogen in the IR was previously noted due to use of the excitation rates of Park

- In an attempt to reduce the effect of the over-prediction, a merged database of the rates from Huo and Park has been created

- The merged database appears to have mitigated the issue of providing an over-prediction for atomic transitions.

- No atomic hydrogen simulated 


\section{Non-equilibrium Metric}

Absolute Non-Equilibrium Radiance

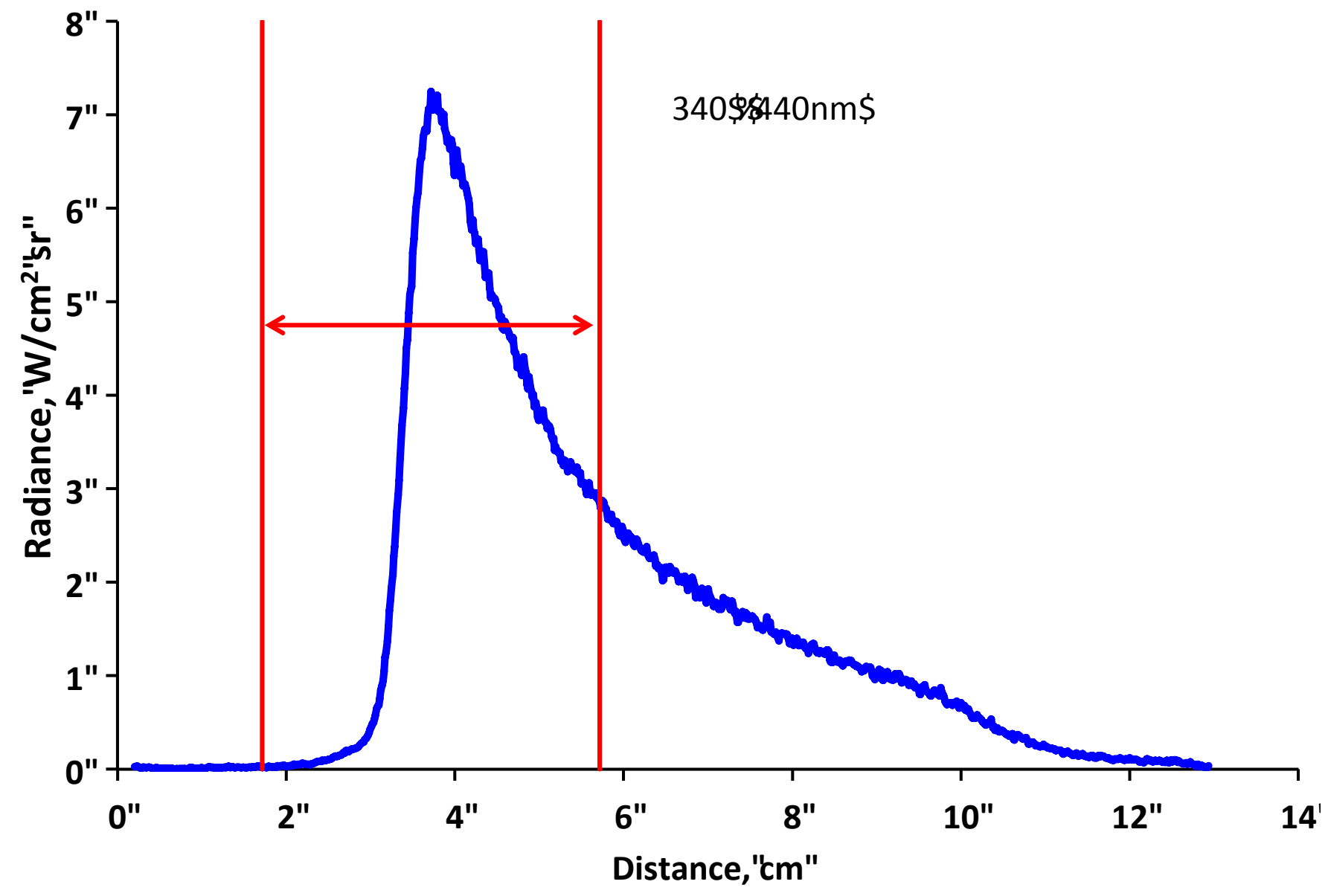

Integrate radiance $\pm 2 \mathrm{~cm}$ either side of shock front. Normalized by shock tube diameter 


\section{Experiment vs Simulations}

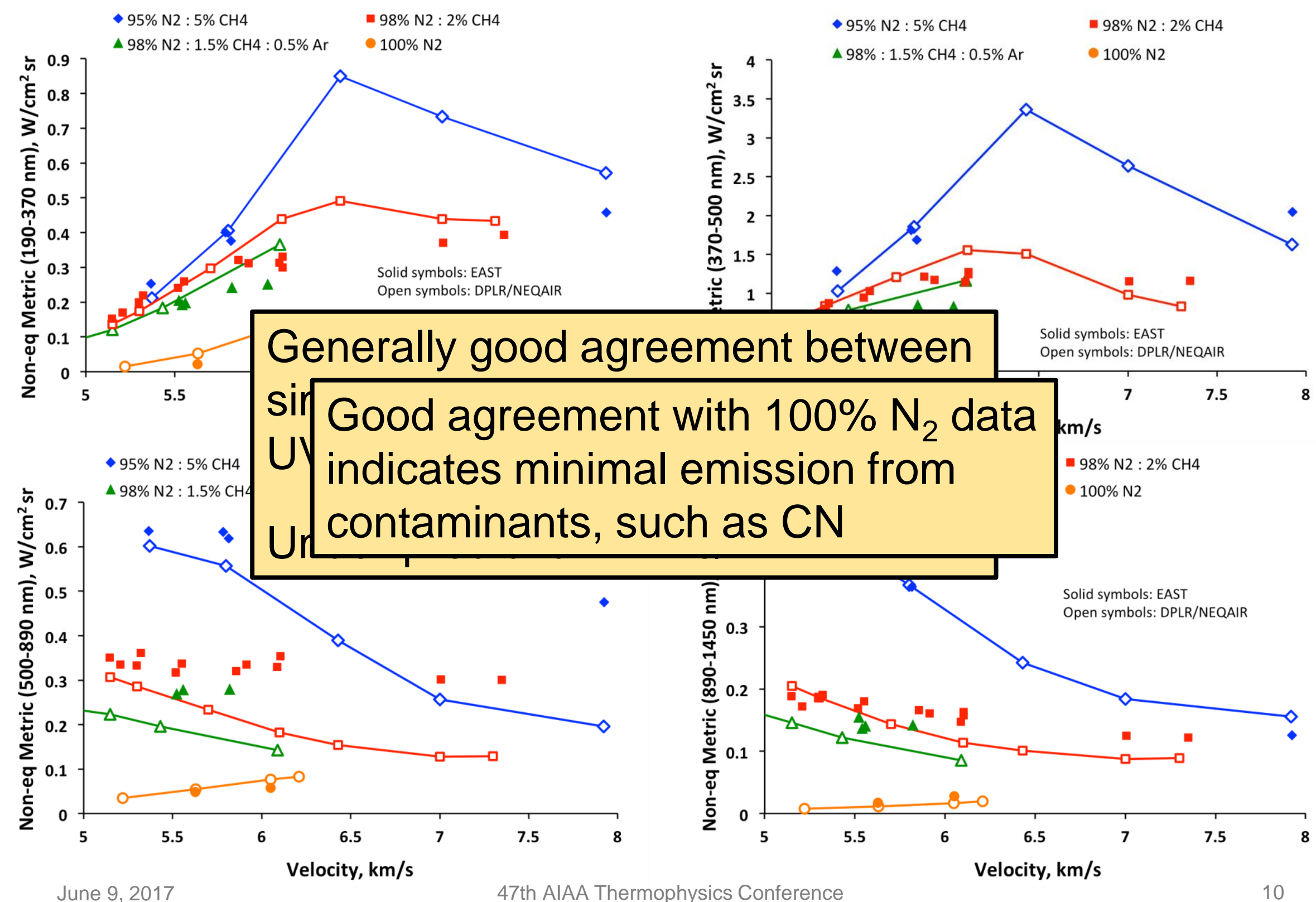




\section{Comparisons To Previous Data: Test $43\left(8.6 \% \mathrm{CH}_{4}\right)$}
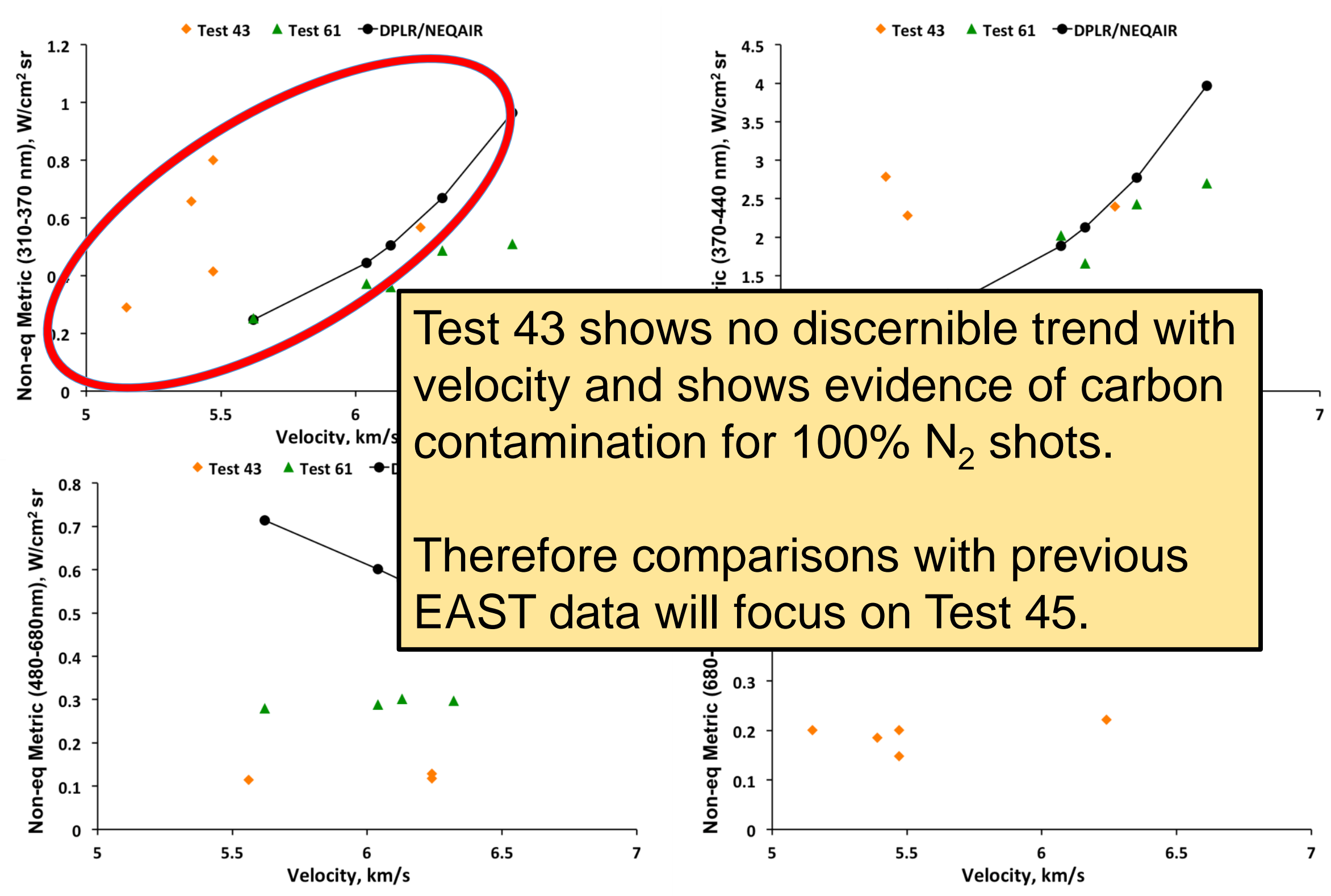

Test $43 \Delta$ Test $61-$ DPLR/NEQAIR 


\section{Comparisons To Previous Data: Test $45\left(2 \% \mathrm{CH}_{4}\right)$}

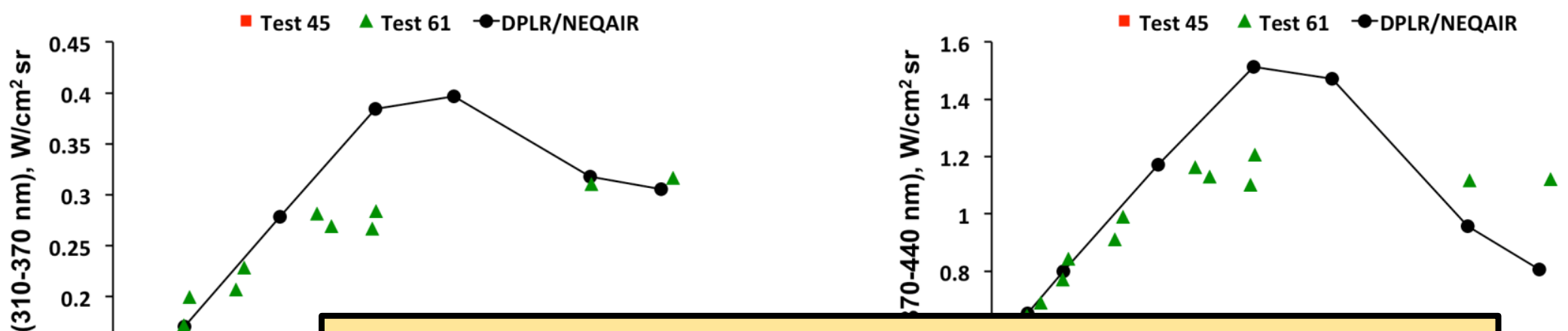

Significantly better agreement between simulations and Test 61 in the UV \& NIR.

Improve agreement with Test 45 for high

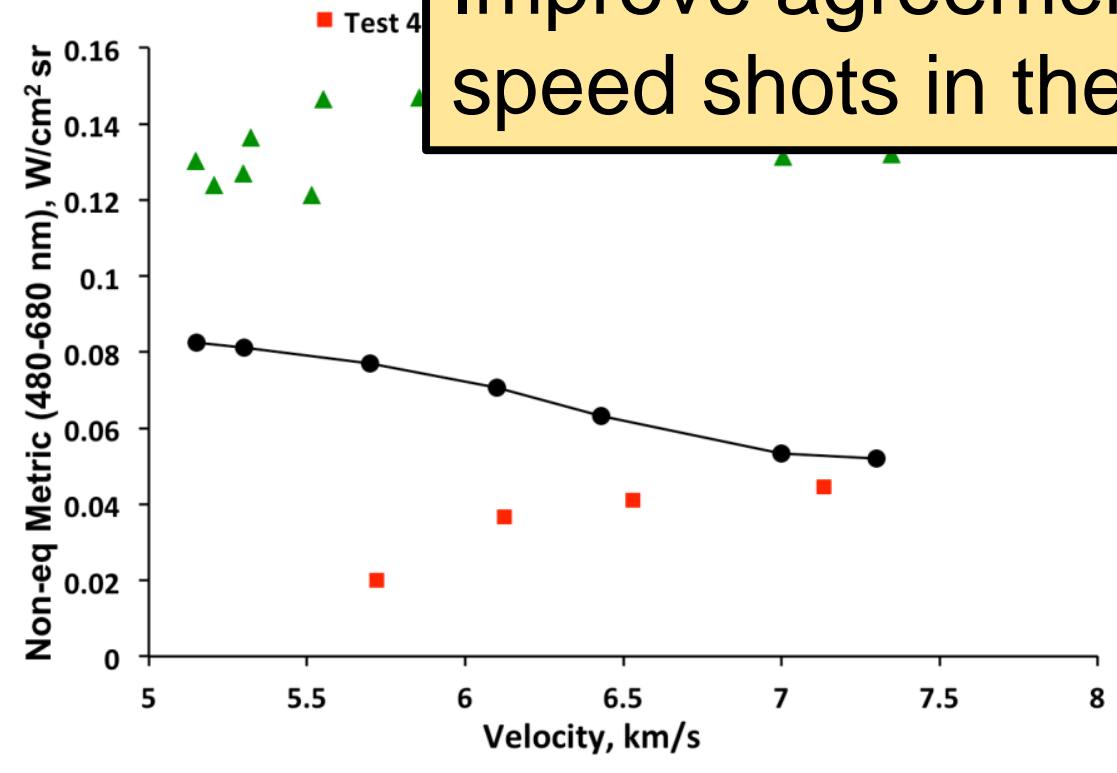

Visible.

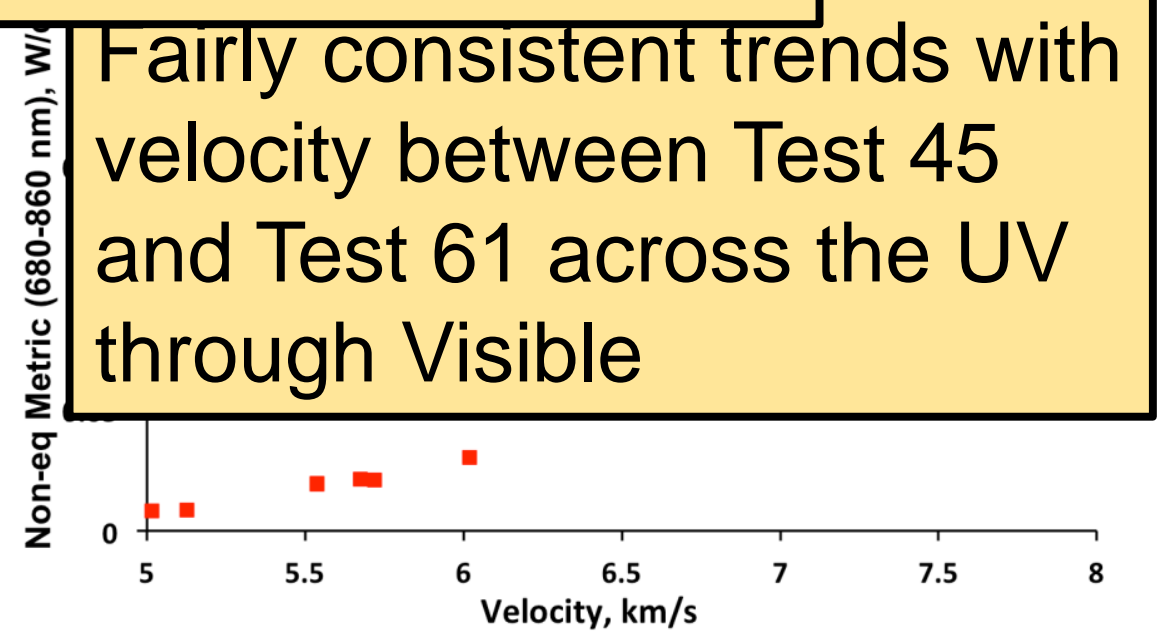




\section{Comparison with Simulations: UV}

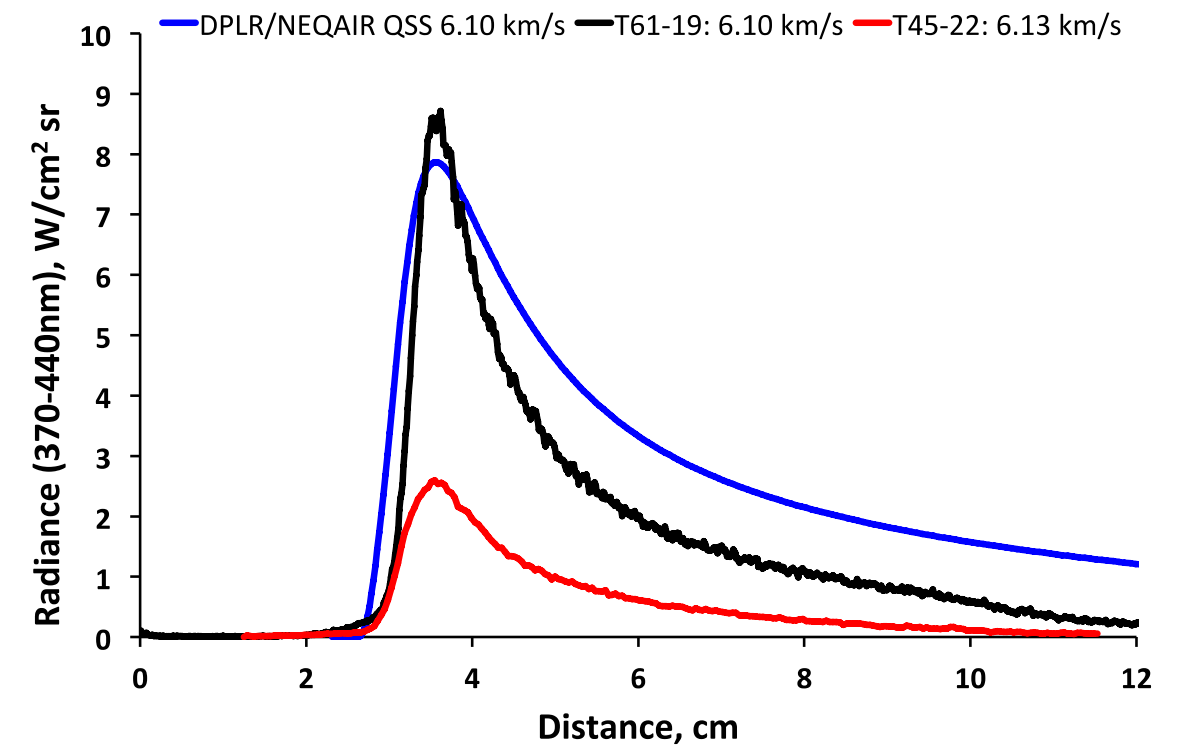

—DPLR/NEQAIR $6.10 \mathrm{~km} / \mathrm{s}$

-T61-19: $6.10 \mathrm{~km} / \mathrm{s}$

-T45-22: $6.13 \mathrm{~km} / \mathrm{s}$

-DPLR/NEQAIR N2X3

立

A

Boltzmann provides significant over-prediction. QSS could be underpredicting $\mathrm{CN}(\mathrm{B})$ population.
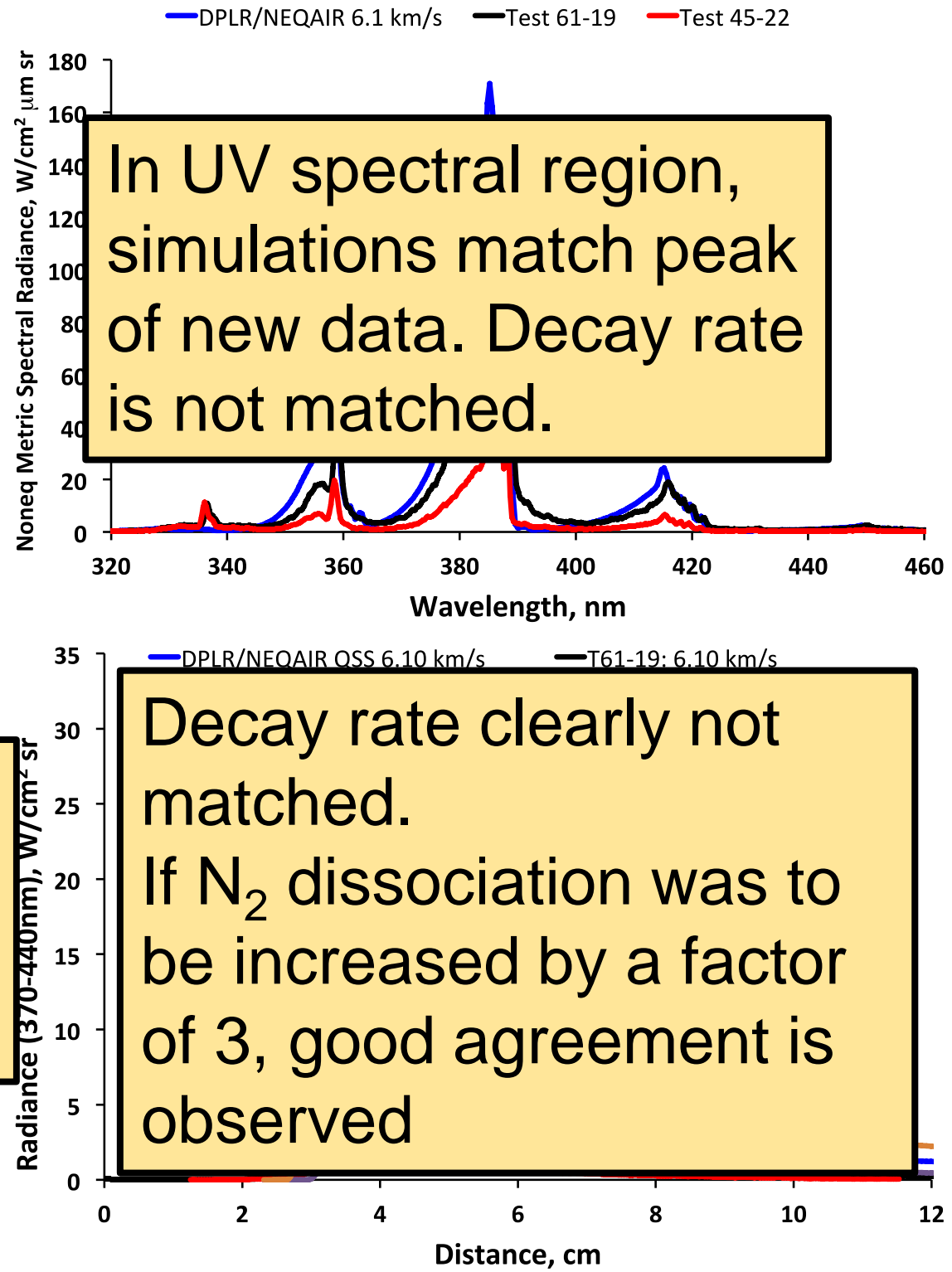


\section{Comparison with Simulations: Red}
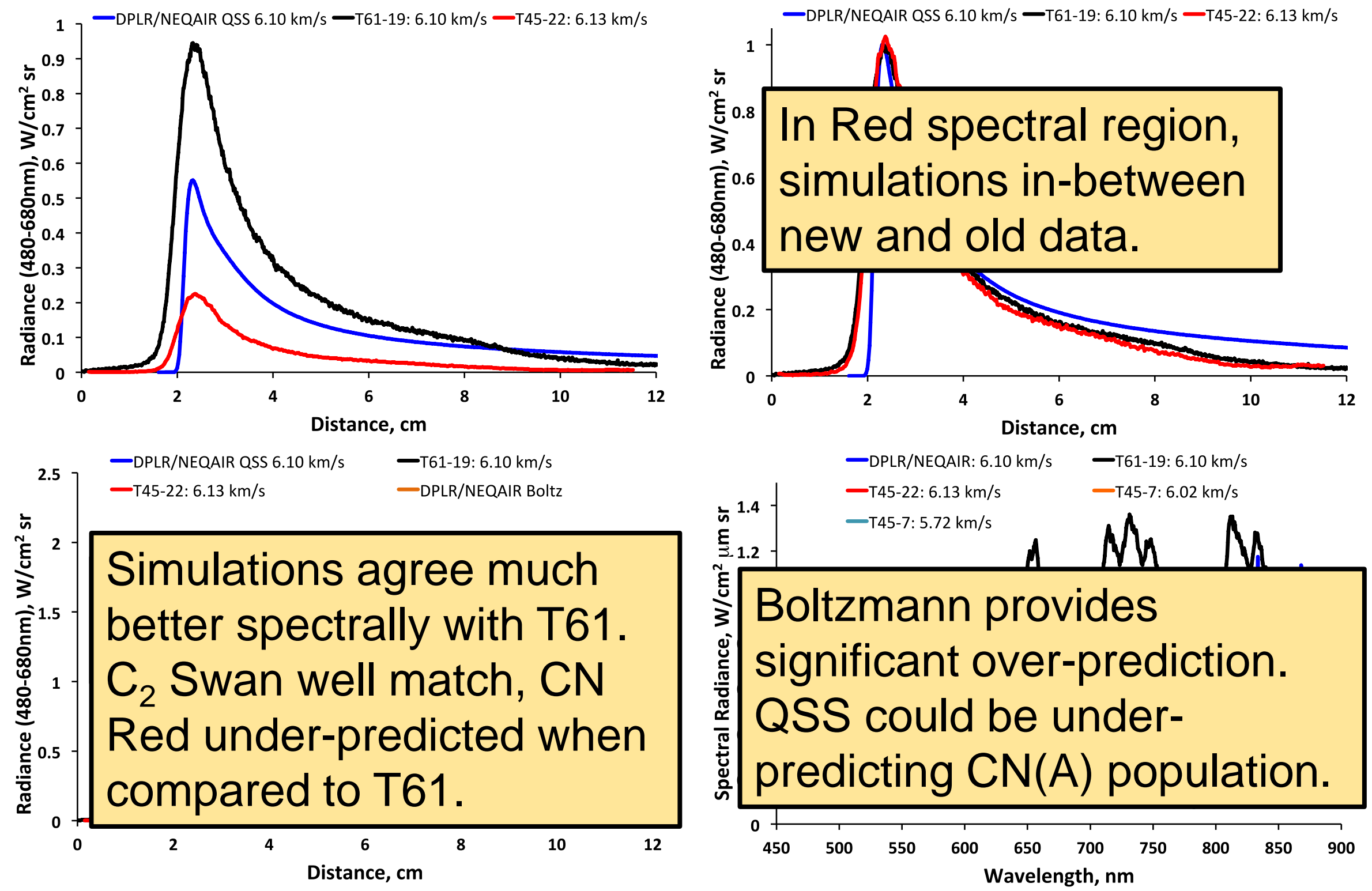


\section{Validation of Calibration}

-Test 62-20 —CEA/NEQAIR

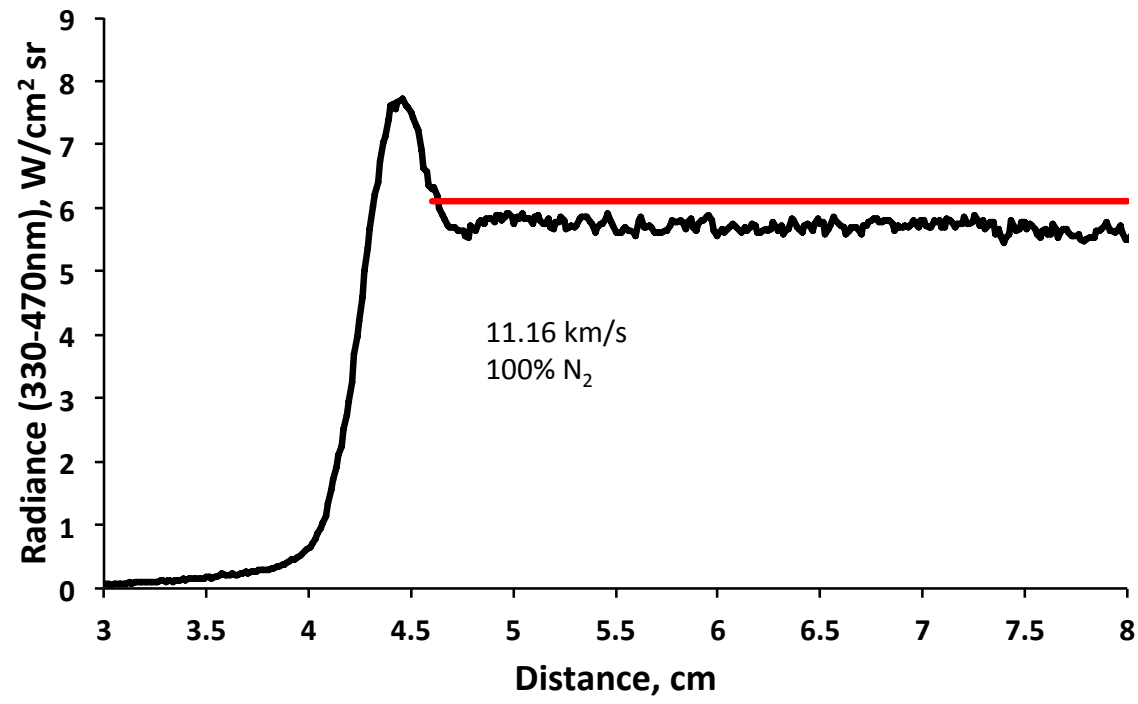

-Test 62-20 -CEA/NEQAIR

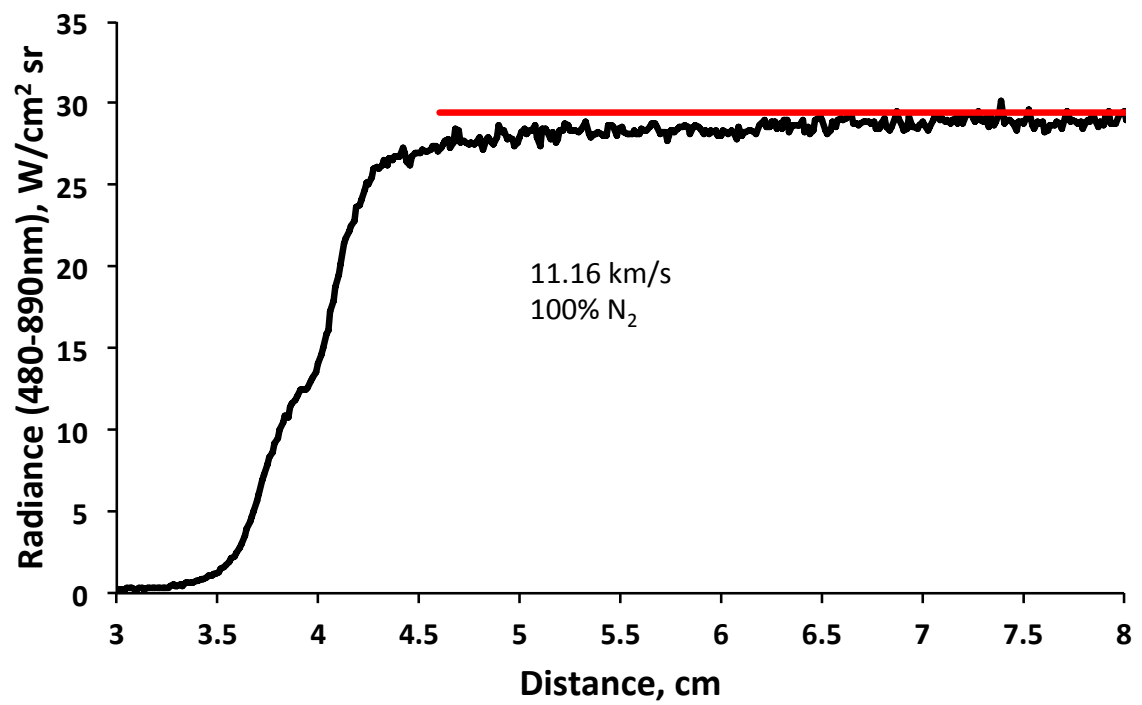

- Tests were conducted to confirm radiance calibration

- Experiments performed in pure nitrogen at high velocity to ensure equilibrium

- Plots cover spectral range corresponding to CN Violet, CN Red $\& \mathrm{C}_{2}$ Swan

Excellent agreement between EAST and equilibrium:

Calibration confirmed 


\section{Examination of Discrepancies}




\section{Facility Operation}

- Due to the significant discrepancies between the Test 61 and Test 45 data, shots were performed removing some of the facility upgrades.

- Two of the upgrades were focused on in this current testing:

- the method of gas loading and mixing the test gas.

- the use of an oxygen plasma cleaner.

- The current gas loading configuration uses mass flow controls to meter the gas into the facility just prior to the shot. Allows the gas to be mixed dynamically and ratios to be specified with flexibility.

- Tests were tried with manually loading the test gas, using a pre-mixed bottle and removing the $\mathrm{O}_{2}$ plasma cleaner 


\section{Facility Operation}

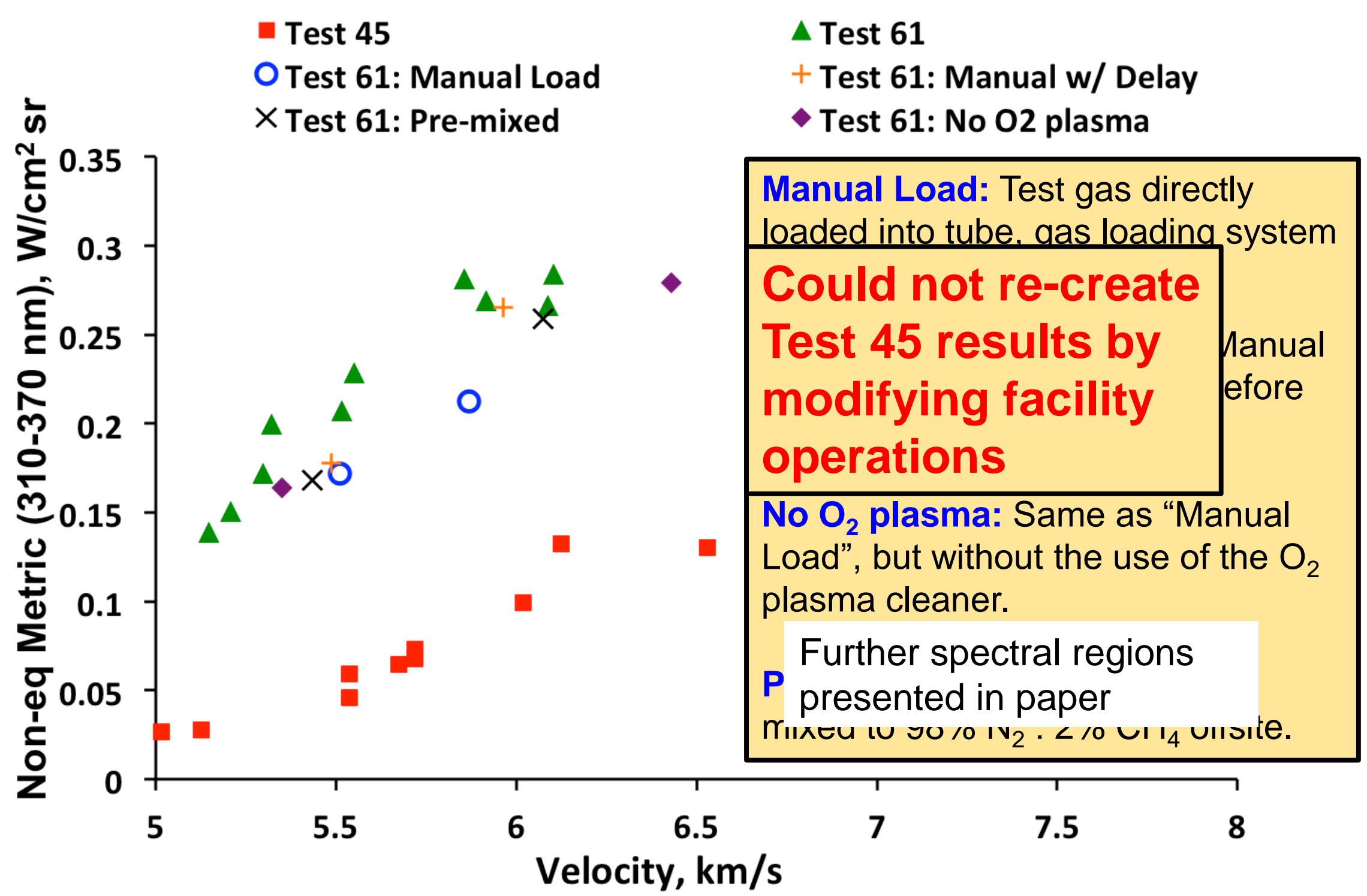




\section{Contamination}

- Previous reports have identified potential calibration and contamination issues with pre-upgrade EAST results.

- It has been reported from test campaigns in X2 that a small amount of air leakage occurs after isolating the vacuum pumps and before firing the test.

- This amount of air might initially appear small, as it is mostly nitrogen, as is the atmosphere of Titan.

- However the addition of oxygen can be very significant as it will react with the $\mathrm{CH}_{4}$ to form $\mathrm{CO}$ and thereby reduce the carbon available to form $\mathrm{CN}$.

- Equilibrium simulations of these shock conditions show that a small amount of oxygen (1.4\%) can reduce the equilibrium CN number density by as much as a factor of two.

- Therefore, air was deliberately added to the EAST test gas to assess this effect. 


\section{Contamination}

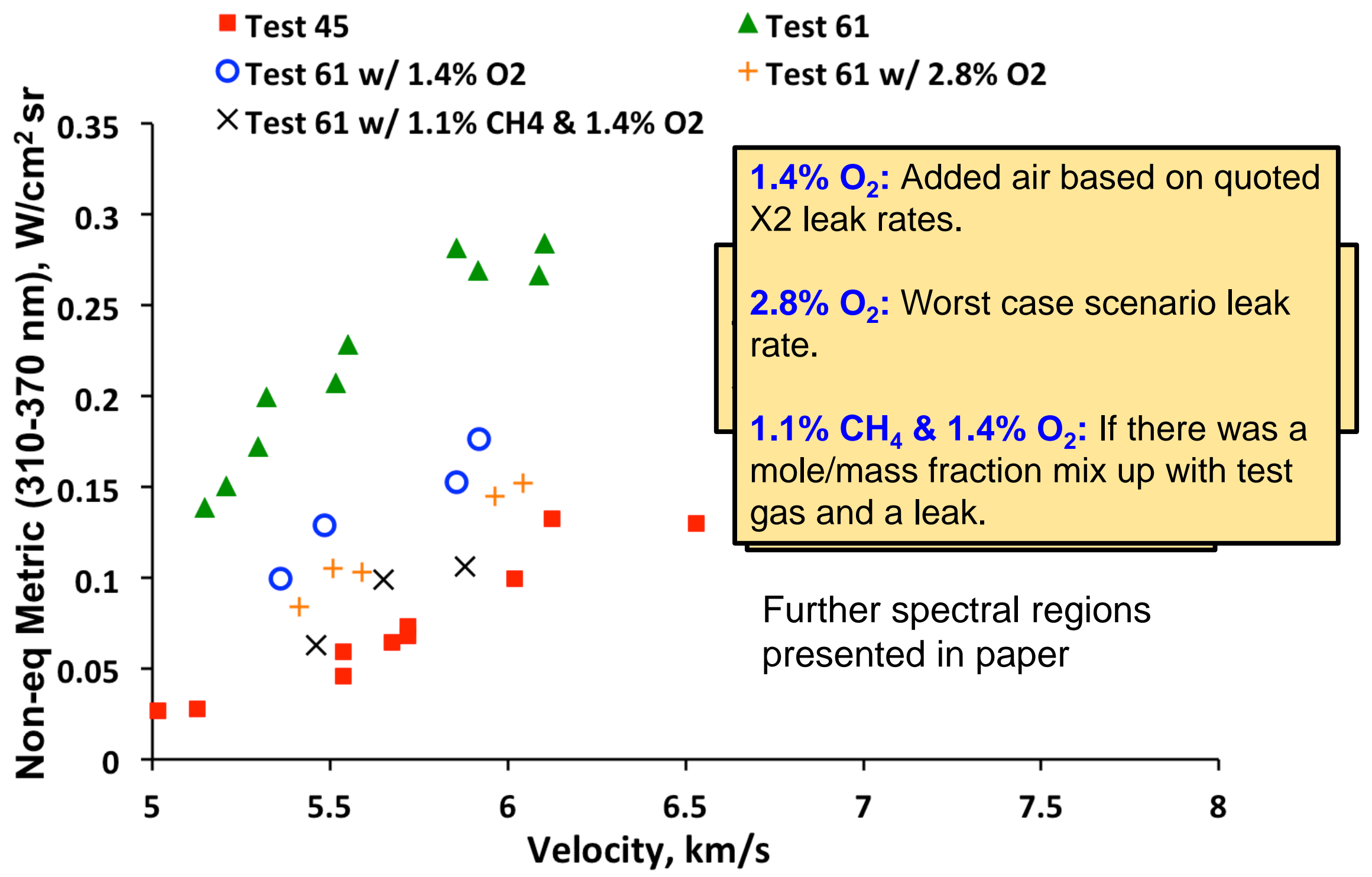




\section{Comparisons To Previous Data: X2, Tests 43 \& 45}

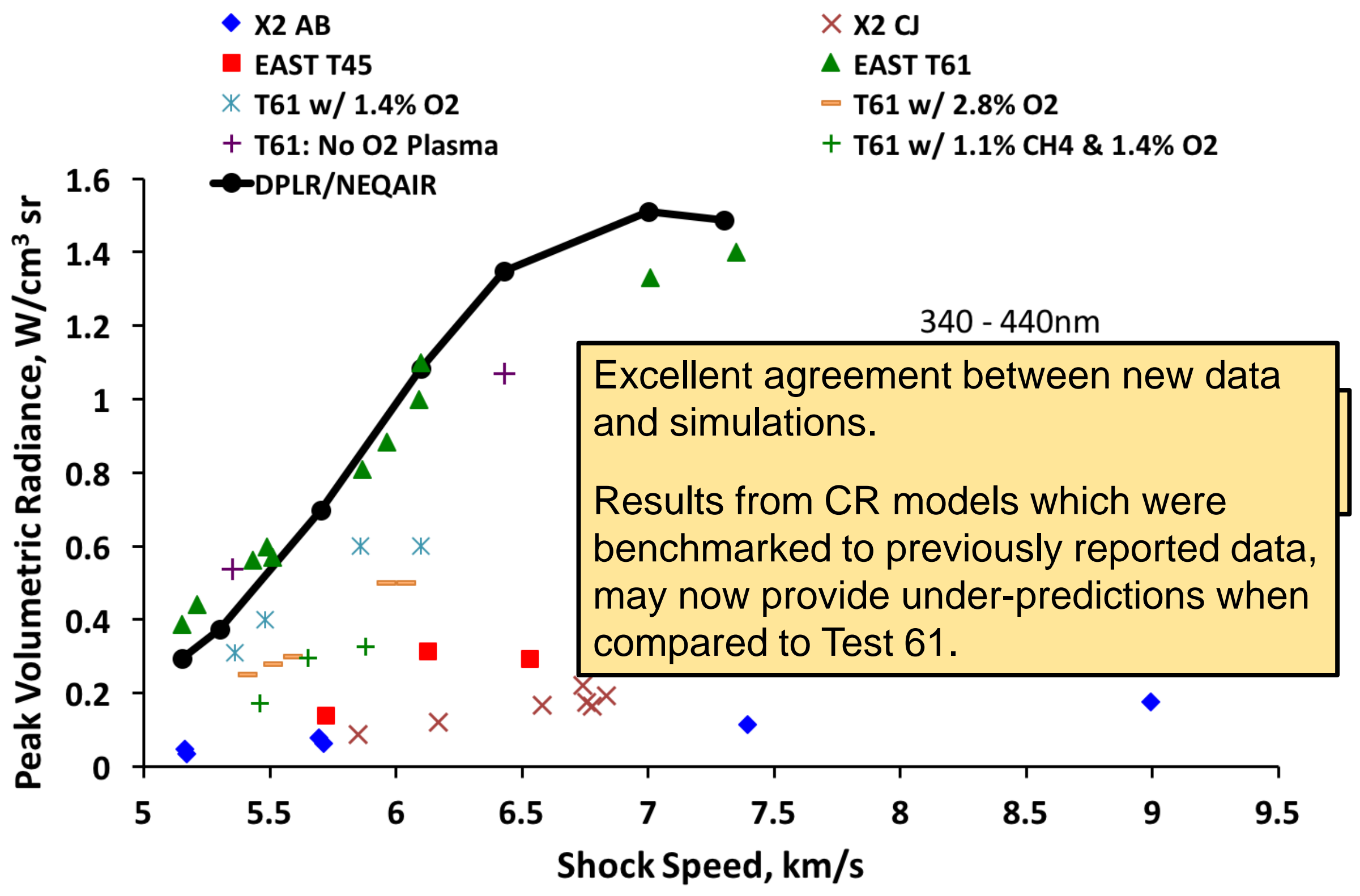




\section{Validation Data}

- The final section of the paper (and the appendix) detail selected experiments from EAST Test 61 for use in future model validation and experimental comparison.

- These selected data sets were chosen based on consistency with other results from Test 61 combined with good experimental characteristics, such as test time.

- The spatially and spectrally resolved radiance is shown for a variety of conditions. 


\section{Validation Data}
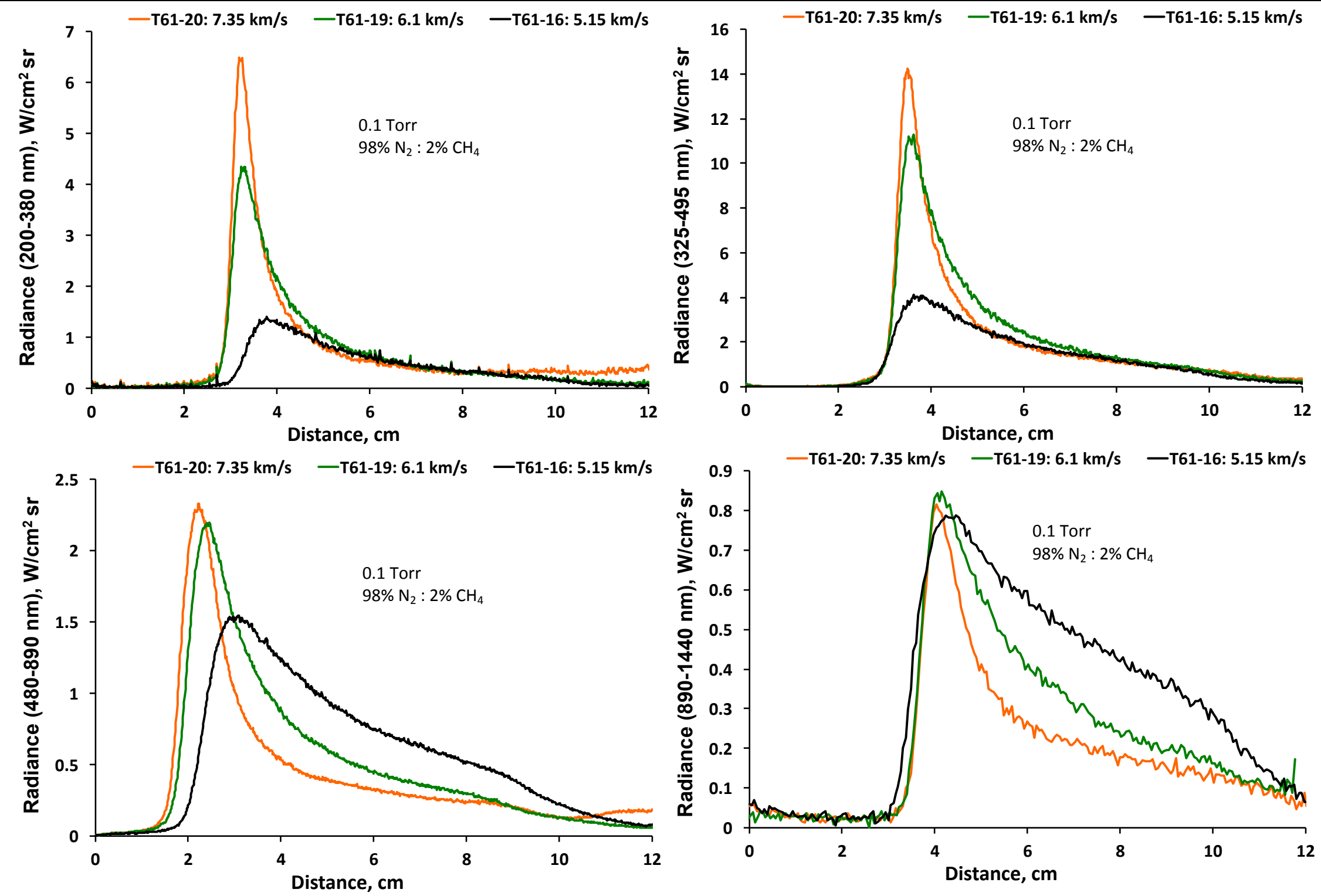


\section{Validation Data}
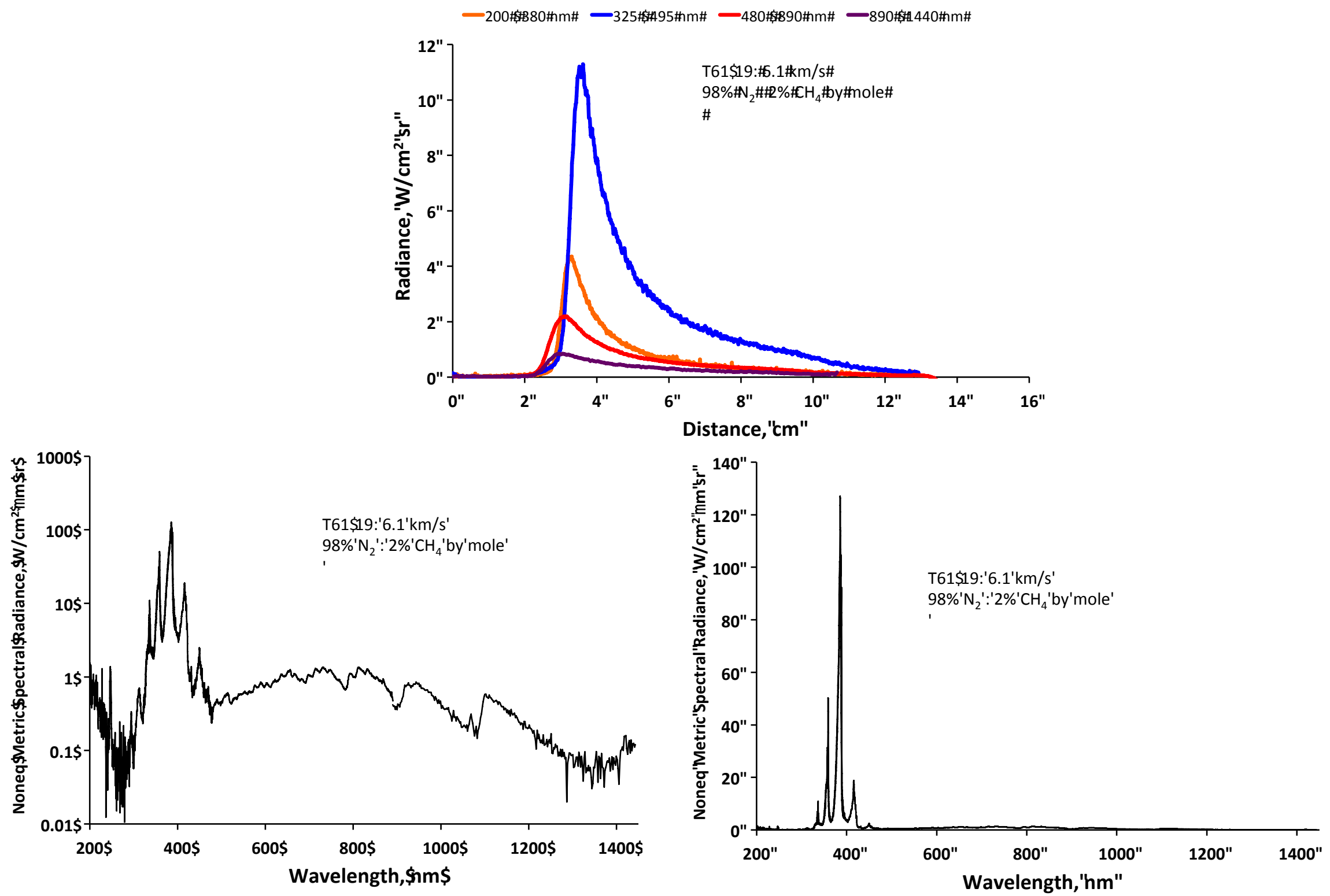


\section{Conclusions}

- Recent measurements for Titan entry made in EAST, known as Test 61

- Results are available at https://data.nasa.gov/

- Test 61 has been compared to previous EAST (T43 \& T45) and X2 campaigns

- Test 61 shows significantly larger radiance

- Generally very good agreement between simulations and the new EAST data

- In particular, peak CN Violet and decay of CN Red very well matched

- Work to be done to resolve discrepancies identified for modelling of $C N(A)$ \& $C N(B)$

- Substantial effort undertaken to understand reasons for discrepancy in results

- The discrepancy can seemingly only be explained by a combination of multiple effects, including contaminants in the test gas used in previous experiments, radiance calibration issues and inaccuracies in shock speed measurements.

- Specific Test 61 experiments have been selected as sources for future code validation studies and facility-to-facility comparisons.

It is recommended that the previously reported Titan entry data be replaced with the current results. 


\section{Questions?}


National Aeronautics and Space Administration

Ames Research Center

Entry Systems and Technology Division 


\section{Validation Data}
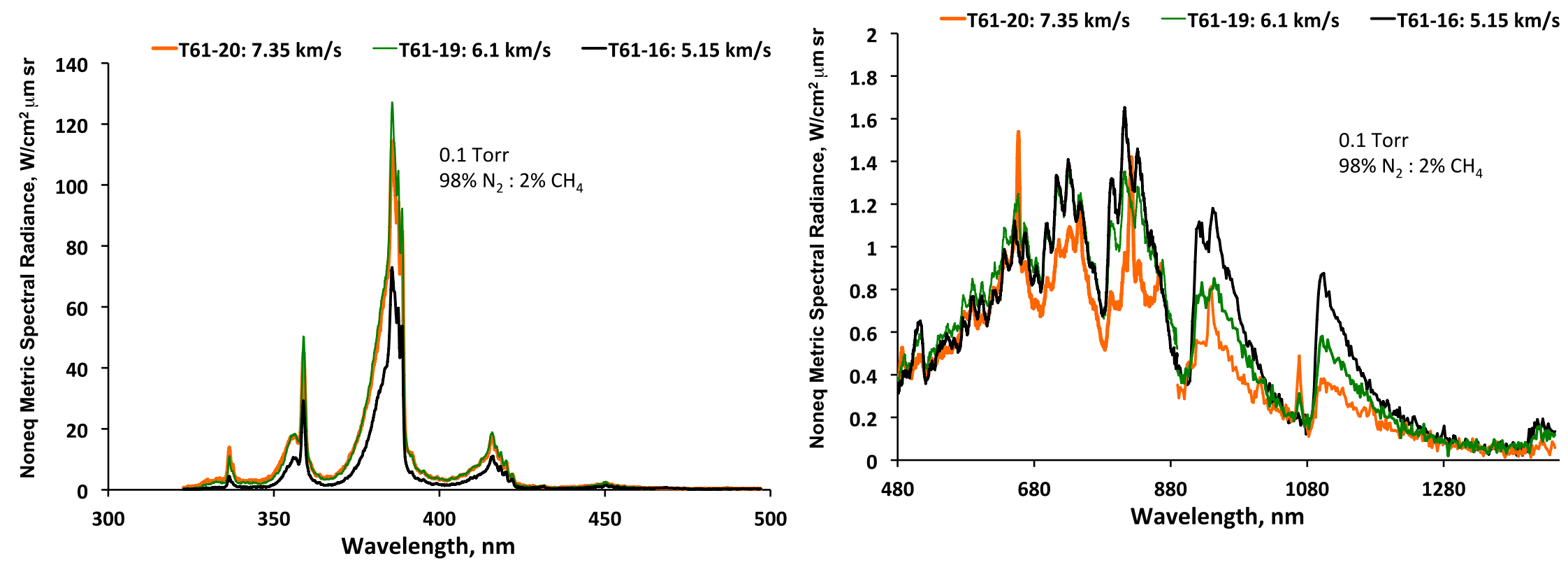


\section{Development of Non-equilibrium Metric}

- Many insights gained by comparing equilibrium radiance vs velocity trends between simulations and experiments.

- For non-equilibrium, it is not clear that 1 metric can represent all aspects of the flow. Ideally, the metric would be:

- Independent of experimental parameters (such as gate width and spatial resolution).

- Applicable to a wide range of conditions.

- Easily comparable to simulation results.

- Consistent with limitations of test time in the facility. 